\title{
Glutathione depression by dihydropyrazine derivative
}

\author{
Shinji Takechi', Shigeru Ito², Nobuhiro Kashige ${ }^{3}$, Takumi Ishida ${ }^{1}$ \\ and Tadatoshi Yamaguchi ${ }^{1}$ \\ ${ }^{1}$ Faculty of Pharmaceutical Sciences, Sojo University, Ikeda 4-22-1, Kumamoto 860-0082, Japan \\ 2Institute of Biomaterials and Bioengineering, Tokyo Medical and Dental University, 2-3-10, Kanda-Surugadai, \\ Chiyoda-ku, Tokyo 101-0062, Japan \\ ${ }^{3}$ Faculty of Pharmaceutical Sciences, Fukuoka University, 8-19-1, Nanakuma, Jonan-ku, Fukuoka 814-0180, Japan
}

(Received November 16, 2010; Accepted December 24, 2010)

\begin{abstract}
Dihydropyrazine (DHP), which is formed by nonenzymatic glycation, generates various radical species that lead to DNA damage and enzyme inhibition. In this study, we examined the reaction between DHP derivatives and glutathione (GSH). DHP exposure caused more intense growth inhibition of a GSH-deficient mutant Escherichia coli strain compared with the wild-type strain. DHP-exposed mouse fibroblasts showed a decrease in the cellular GSH level. The obtained data suggested that the reaction of DHP with GSH possibly potentiates cellular stress via the depletion of cellular GSH levels.
\end{abstract}

Key words: Glycation product, Glutathione, Oxidative stress

\section{INTRODUCTION}

Dihydropyrazine (DHP) is produced by the condensation of two molecules of D-glucosamine (Kashige et al., 1995). We previously reported that DHP derivatives generate various radical species and reactive oxygen species (ROS) that cause DNA strand cleavage in vitro (Yamaguchi et al., 1998). ROS can cause oxidative damage to DNA, proteins and lipids in all living organisms, and the resulting oxidative damage may play roles in several human disorders as well as the aging process (Beckman and Ames, 1998). We hypothesized that DHPs, which are presumed to be precursors of pyrazines, would be responsible for certain diseases, because many pyrazine derivatives have been detected in various foods (Maga and Sizer, 1973) and human urine (Zlatkis et al., 1973). However, there is little evidence regarding a causal relationship between DHP and diseases.

Previously, we reported that methyl-substituted dihydropyrazines (Me-DHPs) have DNA strand-breaking activity, inhibit growth and induce mutagenesis in Escherichia coli (Takechi et al., 2004, 2006). Although phenyl-substituted dihydropyrazines (Ph-DHPs) have much higher DNA strand-breaking activity and produces higher levels of radical species than Me-DHPs (Yamaguchi et al., 1999; Kashige et al., 2005), DNA repair-deficient bacteria are less sensitive to Ph-DHPs than to Me-DHPs (Takeda et al., 2005, 2007). In addition, Cyclohexyl-DHP was shown to possess stronger DNA strand-breaking activity than Me-DHPs and PhDHPs (Maruoka et al., 2005; Yamaguchi et al., 2007). Moreover, we demonstrated that DHPs can potentially generate superoxide anions (Takechi et al., 2009), and not only cause breakage of chromosomal DNA leading to mutagenic lesions but also induce damage to cellular proteins such as glyceraldehyde-3-phosphate dehydrogenase (GAPDH) (Takechi et al., 2010).

In the present study, we found that DHPs cause more intense growth inhibition of glutathione (GSH)-deficient bacteria compared with wild-type bacteria. In addition, we observed a significant loss of GSH in DHP-exposed cultured cells. Our present findings suggest DHP may enforce cellular stress via the depletion of cellular GSH levels.

\section{MATERIALS AND METHODS}

\section{Synthesis of DHP derivatives}

3-Hydro-2,2,5,6-tetramethylpyrazine (Me-DHP-3) and a mixture of two isomers of Cyclohexyl-DHP (Fig. 1), namely 2,3,5,6,7,8-hexahydroquinoxaline (endo-type) and 1,2,3,5,6,7-hexahydroquinoxaline (exo-type), were prepared using a previously described method (Yamaguchi et al., 1998). 


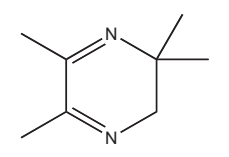

Me-DHP-3 : 3-hydro-2,2,5,6-tetramethylpyrazine

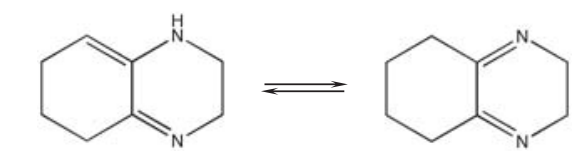

Cyclohexyl-DHP : the mixture of 2,3,5,6,7,8-hexahydroquinoxaline (endo-type) and 1,2,3,5,6,7-hexahydroquinoxaline (exo-type)

Fig. 1. Chemical structures of the DHPs employed in this study.

\section{Bacterial strain}

The E. coli K-12 strain W3110 [LAM-, IN(rrnD$r r n E) 1, r p h-1]$ (Brodie and Reed, 1990) was used as a parental wild-type strain. An isogenic gshA mutant was constructed as follows. The gshA gene from JD27110 [gshA::mini-Tn10(Kan)] was introduced into the W3110 strain by P1-mediated transduction and selection for kanamycin resistance, and subsequently checked by PCR. W3110 was obtained from the E. coli Genetic Resource Center (CGSC, Yale University, New Haven, CT, USA) and JD27110 was obtained from the National BioResource Project (NIG, Shizuoka, Japan).

\section{Growth assay for $E$. colis}

Overnight cultures of $E$. coli were diluted by 100 -fold with fresh M9 glucose medium containing $0.2 \%$ glucose, $1 \mathrm{mM} \mathrm{MgSO}_{4}$ and $1 \mu \mathrm{g} / \mathrm{ml}$ thiamine (Brodie and Reed, 1990), and then grown at $37^{\circ} \mathrm{C}$ until the $\mathrm{OD}_{600}$ reached about 0.1 . The cells were then exposed to various concentrations of DHP, and bacterial growth was monitored by measuring the $\mathrm{OD}_{600}$.

\section{Reaction of DHP with GSH and fluorometrical GSH assay}

The reaction of DHP with GSH was conducted by incubating $10 \mathrm{mM}$ GSH with various concentrations of DHP in $50 \mathrm{mM}$ sodium phosphate buffer $(\mathrm{pH} 7)$ at $37^{\circ} \mathrm{C}$ for $1 \mathrm{hr}$. The amount of GSH was measured fluorometrically using a previously described method (Hissin and Hilf, 1976). Briefly, the assay mixture comprised $1.8 \mathrm{ml}$ of $0.1 \mathrm{M}$ phosphate buffer $(\mathrm{pH} 8.0)$ containing $5 \mathrm{mM}$ EDTA, $0.1 \mathrm{ml}$ of $1 \mathrm{mg} / \mathrm{ml} o$-phthalaldehyde and $0.1 \mathrm{ml}$ of sample. The fluorescence intensity at $420 \mathrm{~nm}$ was determined after activation at $350 \mathrm{~nm}$.

\section{Cell culture}

3T3-L1 murine fibroblasts were obtained from RIKEN BRC (RIKEN, Ibaraki, Japan) and maintained in highglucose Dulbecco's modified Eagle's medium (Gibco BRL, Carlsbad, CA, USA) supplemented with 10\% fetal calf serum (MP Biomedicals, Irvine, CA, USA) and penicillin/streptomycin at $37^{\circ} \mathrm{C}$ in a $5 \% \mathrm{CO}_{2}$-enriched and humidified atmosphere.

\section{Preparation of DHP-exposed cell extracts and total GSH assay}

Confluent 3T3-L1 cells were washed with phosphatebuffered saline (PBS), resuspended in an equal volume of PBS and exposed to various concentrations of DHP at $37^{\circ} \mathrm{C}$ for $1 \mathrm{hr}$. The DHP-exposed cells were washed with PBS, resuspended in an equal volume of cold sulfosalicylic acid (5\%, w/v in distilled water) and subjected to two freeze/thaw cycles. After centrifugation at 14,000 rpm for $10 \mathrm{~min}$, the supernatant was separated and stored at $-80^{\circ} \mathrm{C}$ until analysis. Measurements of total GSH were performed using a Total Glutathione Quantification Kit (Dojin, Tokyo, Japan) according to the manufacturer's instructions. The changes in absorbance at $405 \mathrm{~nm}$ were measured and the concentrations of GSH were calculated relative to a standard curve.

\section{Statistical analysis}

Statistical significance was determined by Williams' multiple comparison test, and a $P$-value less than 0.05 was considered significant $(P<0.05)$.

\section{RESULTS AND DISCUSSION}

We recently reported that DHP derivatives caused inhibition of GAPDH and that SH-compounds suppressed the inhibition of GAPDH by DHP (Takechi et al., 2010). Therefore, we examined the interaction between DHP and $\mathrm{GSH}$, which is the major intracellular thiol compound in organisms. By using two DHP derivatives, Me-DHP-3 and Cyclohexyl-DHP, we investigated the effects of the DHP derivatives on E. coli growth. Fig. 2 shows the growth curves of the wild-type strain ( $w t)$ and the isogenic GSHdeficient strain $(g s h A)$ during exposure to various concentrations of Me-DHP-3 or Cyclohexyl-DHP. Me-DHP-3 hardly affected the growth of the wt strain, but slightly inhibited the growth of the gshA strain. CyclohexylDHP caused modest growth inhibition of the $w t$ strain and conspicuous growth inhibition of the gshA strain. These results suggest that GSH suppresses the DHP-mediated bacteriostatic activity.

When interaction DHP with GSH is direct, DHP caus- 
Dihydropyrazine induces glutathione depletion
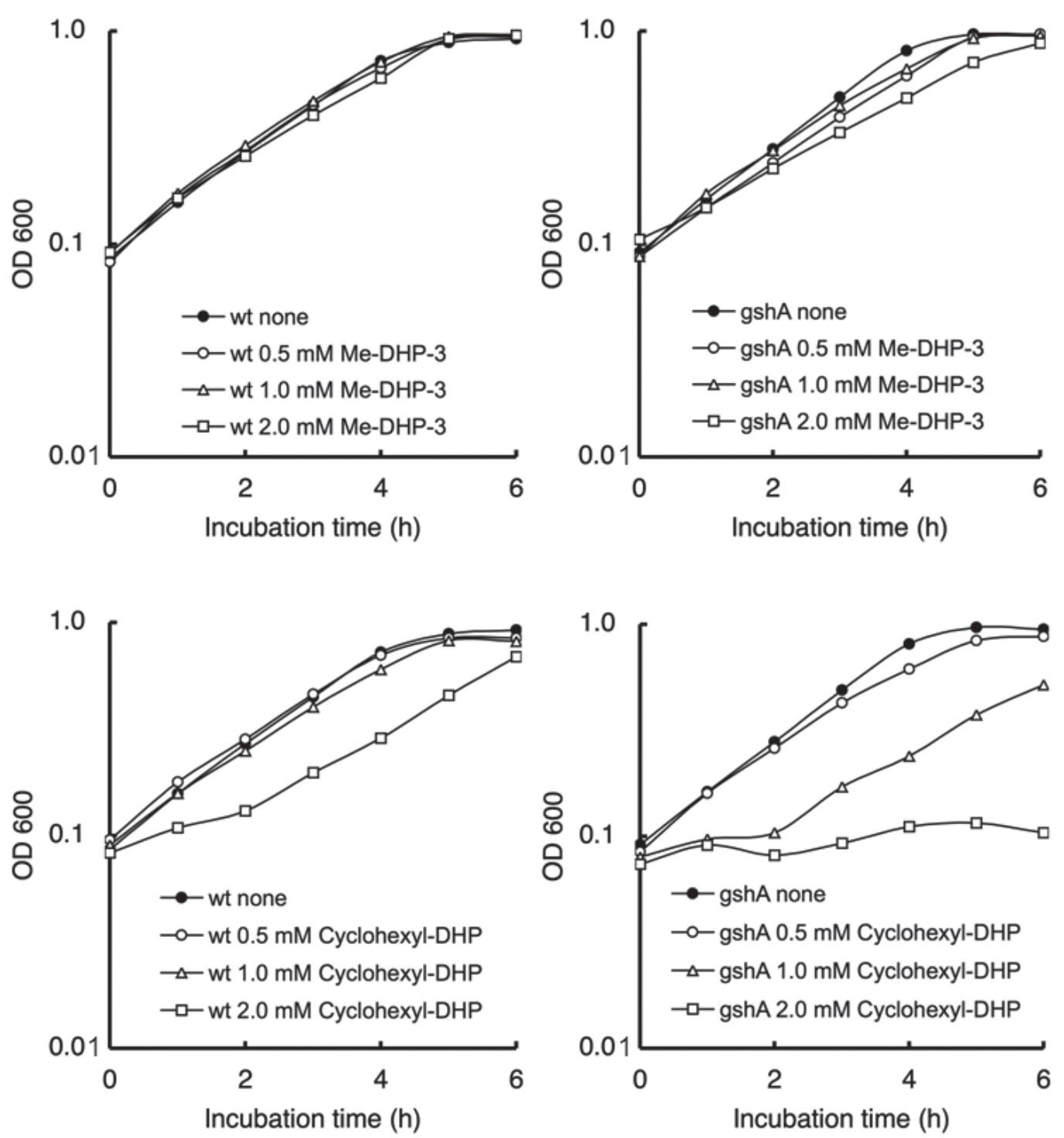

Fig. 2. Inhibition of bacterial growth by DHPs. Various concentrations of Me-DHP-3 or Cyclohexyl-DHP were added to exponentially growing cultures of $w t$ and an isogenic gshA. Growth was monitored at 1-hr intervals by measuring the optical density at $600 \mathrm{~nm}$. The data points reflect the mean values obtained from at least three independent experiments and the error bars show the standard deviations (error bars are not visible on all markers).

es GSH consumption. Thus, we investigated whether DHP would decrease GSH levels in vitro and in vivo. As shown in Fig. 3, when $10 \mathrm{mM}$ GSH was incubated with 0-10 mM DHP in $50 \mathrm{mM}$ sodium phosphate buffer (pH 7) at $37^{\circ} \mathrm{C}$ for $1 \mathrm{hr}$, both Me-DHP-3 and CyclohexylDHP showed a similar dose-dependent decrease of GSH. When 3T3-L1 fibroblasts were exposed to 0-2 mM DHP in PBS at $37^{\circ} \mathrm{C}$ for $1 \mathrm{hr}$, both Me-DHP-3 and CyclohexylDHP also showed a similar dose-dependent decrease of total GSH (Fig. 4). These results suggest that GSH suppressed the DHP causes GSH consumption in vitro and in vivo.

GSH, which acts as the antioxidant, is the major lowmolecular-mass thiol in living organisms (Bhattacharya et al., 1955). It is demonstrated that GSH depletion results in elevated cytotoxicity (Mitchell and Russo, 1987). GSH possesses the thiol group of cysteine donating an electron to other unstable molecules, such as ROS. GSH 
S. Takechi et al.

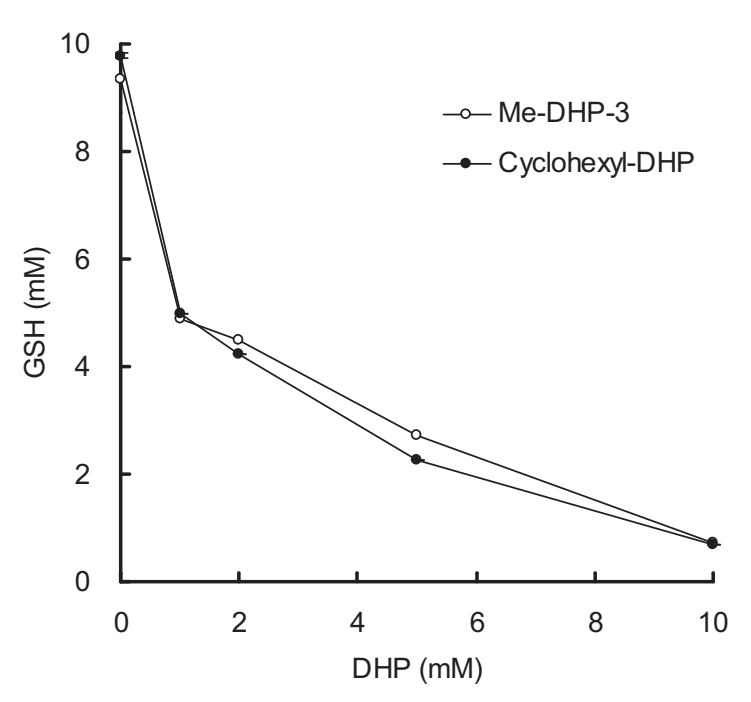

Fig. 3. Effects of DHPs on the GSH levels in vitro. The reaction of DHP with GSH was conducted by incubating 10 $\mathrm{mM}$ GSH with various concentrations of Me-DHP-3 or Cyclohexyl-DHP in $50 \mathrm{mM}$ sodium phosphate buffer (pH 7) at $37^{\circ} \mathrm{C}$ for $1 \mathrm{hr}$. The amount of GSH was measured fluorometrically. The fluorescence intensity at $420 \mathrm{~nm}$ was determined after activation at $350 \mathrm{~nm}$. The data points reflect the mean values obtained from three independent experiments and the error bars show the standard deviations (error bars are not visible on all markers).

itself becomes reactive, and then reactive GSH can react with another reactive GSH to form glutathione disulfide (GSSG) or can react with other reactive molecule (Blair, 2006). GSSG can be readily reverted to GSH by glutathione reductase (Meister and Anderson, 1983).

As compared with $w t$ bacteria, gshA bacteria were more sensitive to DHP in growth assay. In addition, Cyclohexyl-DHP showed stronger bacteriostatic activity than that of Me-DHP-3 (Fig. 2). Consistent with this, in our previous study, Cyclohexyl-DHP exhibited more potent inhibition of GAPDH than that of methyl derivative of DHP (Takechi et al., 2010). Regarding GSH consumption, however, there were no apparent differences in reactivity with GSH between Cyclohexyl-DHP and MeDHP-3 (Figs. 3 and 4). These observations suggested that other thiol-containing molecules besides GSH, such as protein thiols, might be modified by DHP.

ROS are generated by environmental factors, such as ionizing radiation and chemicals, as well as during cellular metabolic processes such as mitochondrial electron transport, immune responses, and the cellular redox sys-

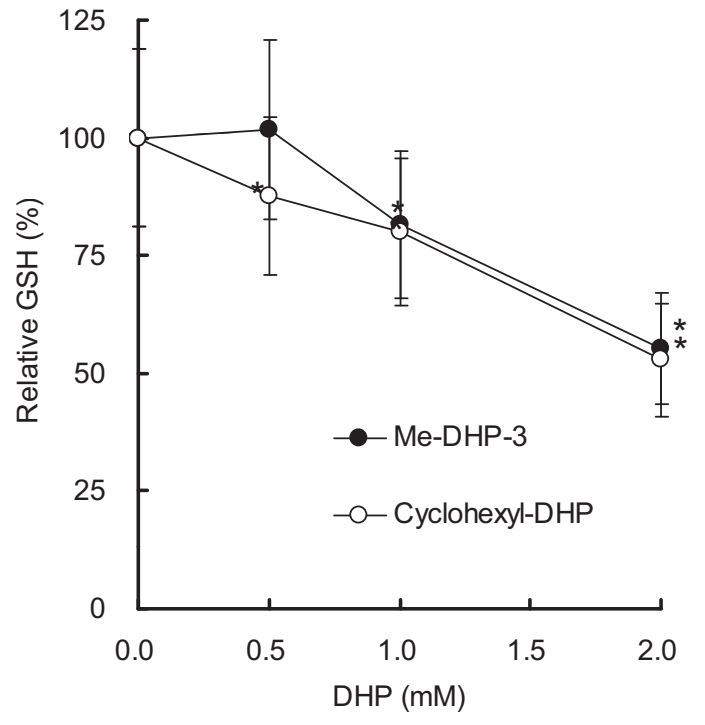

Fig. 4. Effects of DHPs on the cellular GSH levels. 3T3-L1 murine fibroblasts were exposed to various concentrations of Me-DHP-3 or Cyclohexyl-DHP for $1 \mathrm{hr}$, followed by the preparation of cell-free extracts. Measurements of GSH were performed using a Total Glutathione Quantification Kit. Each concentration is given as a percentage of the initial concentration. The data points reflect the mean values obtained from three independent experiments and the error bars show the standard deviations. ${ }^{*} p<0.05$, significantly different from control.

tem (Cance et al., 1979; Weizman and Gordon, 1990). Although various antioxidants protect cells against oxidative stress, ROS can damage subcellular components, thereby resulting in apoptosis, cancer, neurodegenerative diseases, chronic inflammatory diseases, cardiovascular diseases, and aging (Beckman and Ames, 1998; Weizman and Gordon, 1990). In our previous report we showed how DHP strongly produced radicals, suggesting that DHP reached an unstable state by releasing an electron, subsequently generating radicals (Kashige et al., 2005). Our present study provides evidence that DHP, as a generator of ROS, oxidize GSH, resulting in the depletion of cellular GSH levels. On the basis of these observations, we propose that DHP could play a role in disturbances cells' metabolism, like disorders of oxidative-antioxidative balance.

\section{ACKNOWLEDGMENTS}

We thank Takahiro Fujimoto, Mami Mamitsuka, Sayaka Taki, Naoki Yahisa, Rise Yamamoto, Ayumi Yamasaki 
Dihydropyrazine induces glutathione depletion

and Ryousuke Yamashita for their assistance.

\section{REFERENCES}

Beckman, K.B. and Ames, B.N. (1998): The free radical theory of aging matures. Physiol. Rev., 78, 547-581.

Bhattacharya, S.K., Robson, J.S. and Stewart, C.P. (1955): The determination of glutathione in blood and tissues. Biochem. J., 60, 696-702.

Blair, I.A. (2006): Endogenous glutathione adducts. Curr. Drug Metab., 7, 853-872.

Brodie, A.E. and Reed, D.J. (1990): Cellular recovery of glyceraldehyde-3-phosphate dehydrogenase activity and thiol status after exposure to hydroperoxides. Arch. Biochem. Biophys., 276, 212-218.

Cance, B., Sies, H. and Boveris, A. (1979): Hydroperoxide metabolism in mammalian organs. Physiol. Rev., 59, 527-605.

Hissin, P.J. and Hilf, R. (1976): A fluorometric method for determination of oxidized and reduced glutathione in tissues. Anal. Biochem., 74, 214-226.

Kashige, N., Yamaguchi, T., Mishiro, N., Hanazono, H., Miake, F. and Watanabe, K. (1995): Possible involvement of dihydrofructosazine in the DNA breaking activity of D-glucosamine. Biol. Pharm. Bull., 18, 653-658.

Kashige, N., Takeuchi, T., Matsumoto, S., Takechi, S., Miake, F. and Yamaguchi, T. (2005): Radical species in DNA strand-cleavage caused by dihydropyrazines. Biol. Pharm. Bull., 28, 419-423.

Maga, J.A. and Sizer, C.E. (1973): Pyrazines in foods. J. Agric. Food Chem., 21, 22-30.

Maruoka, H., Kashige, N., Miake, F. and Yamaguchi, T. (2005): Synthesis of new dihydropyrazines with DNA strand-breakage activity. Chem. Pharm. Bull., 53, 1359-1361.

Meister, A. and Anderson, M.E. (1983): Glutathione. Annu. Rev. Biochem., 65, 83-100.

Mitchell, J.B. and Russo, A. (1987): The role of glutathione in radiation and drug induced cytotoxicity. Br. J. Cancer, 8, Suppl., 96-104.

Takechi, S., Yamaguchi, T., Nomura,H., Minematsu, T. and Nakayama T. (2004): Growth inhibition and mutagenesis induced in Escherichia coli by dihydropyrazines with DNA strand-cleaving activity. Mutat. Res., 560, 49-55.

Takechi, S., Yamaguchi, T., Nomura, H., Minematsu, T., Adachi, M., Kurata, H. and Kurata, R. (2006): Mutation spectrum induced by dihydropyrazines in Escherichia coli. Biol. Pharm. Bull., 29, 17-20.

Takechi, S., Nakahara, K., Adachi, M. and Yamaguchi, T. (2009): Oxidative stress induced by a dihydropyrazine derivative. Biol. Pharm. Bull., 32, 186-189.

Takechi, S., Nakahara, K. and Yamaguchi, T. (2010): Dihydropyrazine-induced inactivation of glyceraldehyde-3-phosphate dehydrogenase. Biol. Pharm. Bull., 33, 379-383.

Takeda, O., Takechi, S., Katoh, T. and Yamaguchi, T. (2005): The Role of dihydropyrazines in accelerated death of Escherichia coli on addition of copper(II). Biol. Pharm. Bull., 28,1161-1164.

Takeda, O., Takechi, S., Ito S., Omori, H., Katoh, T. and Yamaguchi, T. (2007): Effects of phenyl derivatives of dihydropyrazines with ability to generate radical species on Escherichia coli. Biol. Pharm. Bull., 30, 1663-1667.

Weizman, S.A. and Gordon, L.I. (1990): Inflammation and cancer: role of phagocyte-generated oxidants in carcinogenesis. Blood, 76, 655-663.
Yamaguchi, T., Matsumoto, S. and Watanabe, K. (1998): Generation of free radicals from dihydropyrazines with DNA strand-breakage activity. Tetrahedron. Lett., 39, 8311-8312.

Yamaguchi, T., Eto, M., Harano, K., Kashige, N., Watanabe, K. and Ito, S. (1999): New compounds derived from dihydropyrazines having DNA strand-breakage activity. Tetrahedron, 55, 675-686.

Yamaguchi, T., Ito, S., Kashige, N., Nakahara, K. and Harano, K. (2007): The relationship between the chemical structures of dihydropyrazine derivatives and DNA strand-breakage activity. Chem. Pharm. Bull., 55, 532-536.

Zlatkis, A., Bertsch, W., Lichtenstein, H.A., Tishbee, A., Shunbo, F., Liebich, H.M., Coscia, A.M. and Fleischer, N. (1973): Profile of volatile metabolites in urine by gas chromatography-mass spectrometry. Anal. Chem., 45, 763-767. 